\title{
OPTIMAL CONTROL OF SEMILINEAR PARABOLIC EQUATIONS WITH STATE-CONSTRAINTS OF BOTTLENECK TYPE*
}

\author{
MaÏtine Bergounioux ${ }^{1}$ And Fredi TrÖLtzsch ${ }^{2}$
}

\begin{abstract}
We consider optimal distributed and boundary control problems for semilinear parabolic equations, where pointwise constraints on the control and pointwise mixed control-state constraints of bottleneck type are given. Our main result states the existence of regular Lagrange multipliers for the state-constraints. Under natural assumptions, we are able to show the existence of bounded and measurable Lagrange multipliers. The method is based on results from the theory of continuous linear programming problems.
\end{abstract}

Résumé. Nous étudions des problèmes de contrôle optimal gouvernés par des équations aux dérivés partielles paraboliques semilinéaires. Le contrôle est à la fois distribué et frontière et les contraintes sont ponctuelles sur le contrôle d'une part, et mixtes de type "bottleneck" sur le contrôle et l'état. Sous des hypothèses tout à fait naturelles nous prouvons l'existence de multiplicateurs de Lagrange mesurables et bornés. Nous utilisons pour cela des résultats provenant de la programmation mathématique linéaire, continue.

AMS Subject Classification. 49K20, 90C48, 90C45.

Received July 22, 1998. Revised March 16 and October 7, 1999.

\section{Setting of the Problem}

Let $\Omega \subset \mathbb{R}^{N},(N \geq 2)$ be an open bounded domain with boundary $\Gamma$ and let $T>0$ be given fixed. We consider the following semilinear partial differential equation:

$$
\left\{\begin{aligned}
y_{t}+A y+d(x, t, y) & =u & & \text { in } Q=\Omega \times] 0, T[ \\
\partial_{\nu_{A}} y+b(x, t, y) & =v & & \text { on } \Sigma \\
y(x, 0) & =y_{o}(x) & & \text { in } \Omega
\end{aligned}\right.
$$

where $A$ is a uniformly elliptic differential operator defined below, $\Sigma$ denotes the lateral boundary of $Q$ and $\partial_{\nu_{A}} y$ denotes the outward conormal derivative of $y$ associated with $A$. Let us specify the assumptions on the data:

\footnotetext{
Keywords and phrases: Parabolic equation, optimal control, pointwise state-constraint, bottleneck problem.

* This work was partially supported by EEC, HCM Contract CHRX-CT94-0471.

1 Département de Mathématiques, UMR 6628, Université d'Orléans, BP. 6759, 45067 Orléans Cedex 2, France;

e-mail: Maitine.Bergounioux@labomath.univ-orleans.fr

2 Technical University of Chemnitz, Faculty of Mathematics, 09107 Chemnitz, Germany;

e-mail: f.troeltzsch@mathematik.tu-chemnitz.de
} 
(A1) The boundary $\Gamma$ is of class $C^{2, \beta}$, for some $0<\beta \leq 1$. The operator $A$ is defined by

$$
\begin{gathered}
A y=-\sum_{i, j=1}^{N} \partial_{x_{i}}\left(a_{i j}(x) \partial_{x_{j}} y\right) \text { with } \\
a_{i j}=a_{j i} \in \mathcal{C}^{1, \beta}(\bar{\Omega}), \text { for } i, j=1 \cdots N, \\
\forall x \in \bar{\Omega}, \forall \xi \in \mathbb{R}^{N}, m \sum_{i=1}^{N} \xi_{i}^{2} \leq \sum_{i, j=1}^{N} a_{i j}(x) \xi_{i} \xi_{j} \leq M \sum_{i=1}^{N} \xi_{i}^{2} \text { with } m>0 .
\end{gathered}
$$

(A2)

(i) For every $y \in \mathbb{R}$, the function $d=d(\cdot, \cdot, y)$ is measurable on $Q$. It is supposed to satisfy the following assumption of smoothness:

- for almost every $(x, t) \in Q, d$ is continuously differentiable with respect to $y$.

- There are constants $M_{1}, c_{o}$, and a continuous monotone nondecreasing function $\eta:[0, \infty) \rightarrow \mathbb{R}^{+}$such that

$$
|d(x, t, 0)| \leq M_{1} \text { and } c_{o} \leq d_{y}(x, t, y) \leq M_{1} \eta(|y|) .
$$

(ii) Analogous conditions are imposed on $b=b(x, t, y)$ on $\Sigma \times \mathbb{R}$ with the same constants.

(iii) $y_{o}$ belongs to $\mathcal{C}(\bar{\Omega})$.

We shall denote the real function $d$ and the associated Nemytskii operator $d: y(\cdot) \mapsto d(\cdot, y(\cdot))$ by the same symbol. In other words, we write $d(x, t, y(x, t)):=d(y)(x, t),(x, t) \in Q$, since this will not cause confusion. We consider the optimal control problem

$$
(\mathcal{P}) \quad\left\{\begin{array}{c}
\min J(y, u, v) \\
(y, u, v) \text { satisfies }(1.1) \\
(y, u, v) \in C
\end{array}\right.
$$

where

$$
J(y, u, v)=\frac{\alpha_{Q}}{2}\left\|y-z_{Q}\right\|_{Q}^{2}+\frac{\alpha_{\Omega}}{2}\left\|y(T)-z_{\Omega}\right\|_{\Omega}^{2}+\frac{\alpha_{\Sigma}}{2}\left\|y-z_{\Sigma}\right\|_{\Sigma}^{2}+\frac{\alpha_{u}}{2}\|u\|_{Q}^{2}+\frac{\alpha_{v}}{2}\|v\|_{\Sigma}^{2}
$$

$\|\cdot\|_{S}$ denotes the natural norm of $L^{2}(S)$, and $\alpha_{Q}, \alpha_{\Omega}, \alpha_{\Sigma}, \alpha_{u}, \alpha_{v}$ are nonnegative real constants such that $\alpha_{Q}+\alpha_{\Omega}+\alpha_{\Sigma}+\alpha_{u}+\alpha_{v} \neq 0$. Moreover, $z_{Q} \in L^{\infty}(Q), z_{\Sigma} \in L^{\infty}(\Sigma)$ and $z_{\Omega} \in L^{\infty}(\Omega)$ are given fixed. $C$ is a convex set of constraints of bottleneck type. We consider two kinds of such sets.

- $C_{1}$ is the set of all $(y, u, v) \in C(\bar{Q}) \times L^{\infty}(Q) \times L^{\infty}(\Sigma)$ satisfying the inequalities

$$
\text { (I) }\left\{\begin{array}{c}
0 \leq u, 0 \leq v \\
u \leq c_{Q}+\beta_{Q} y \\
v \leq c_{\Sigma}+\beta_{\Sigma} y
\end{array}\right.
$$

a.e. on $Q$ and $\Sigma$, respectively, where $c_{Q}, \beta_{Q} \in L^{\infty}(Q)$ and $c_{\Sigma}, \beta_{\Sigma} \in L^{\infty}(\Sigma)$ are given nonnegative functions. We assume the existence of $\delta>0$ such that $c_{\Sigma}, c_{Q} \geq \delta>0$ holds a.e. on $Q$ and $\Sigma$, respectively.

- $C_{2}$ is the set of all $(y, u, v) \in C(\bar{Q}) \times L^{\infty}(Q) \times L^{\infty}(\Sigma)$ satisfying

$$
\text { (II) } \quad \begin{cases}0 \leq u \leq u_{b}, 0 \leq v \leq v_{b} & (\text { IIa) } \\ \text { and } & \text { (Ib) }\end{cases}
$$

a.e. on $Q$ and $\Sigma$, respectively, where $u_{b} \in L^{\infty}(Q)$ and $v_{b} \in L^{\infty}(\Sigma)$ are given. 
Remark 1.1. The simple choice for $J$ was taken for convenience. It is quite standard in the context of least squares problems. Any other sufficiently smooth integral functional can be chosen as well. For instance, we refer to the objectives defined in Casas [3] or Raymond and Zidani [5].

We shall denote by $\left(\mathcal{P}_{i}\right)$ the problem $(\mathcal{P})$ under the choice $C=C_{i}, i=1,2$. Let us fix the state-space for $y$ as follows: for $q>N / 2+1$ and $s>N+1$

$$
\mathcal{Y}=\left\{y \in W(0, T) \mid y_{t}+A y \in L^{q}(Q), \partial_{\nu_{A}} y \in L^{s}(\Sigma), y(0) \in \mathcal{C}(\bar{\Omega})\right\},
$$

where the conormal derivative $\partial_{\nu_{A}} y$ is defined according to [5], and

$$
W(0, T)=\left\{y \in L^{2}\left(0, T ; H^{1}(\Omega)\right) \mid y_{t} \in L^{2}\left(0, T ; H^{1}(\Omega)^{\prime}\right)\right\} .
$$

$\mathcal{Y}$ is endowed with the norm

$$
\|y\|_{\mathcal{Y}}=\|y\|_{W(0, T)}+\left\|y_{t}+A y\right\|_{L^{q}(Q)}+\left\|\partial_{\nu_{A}} y\right\|_{L^{s}(\Sigma)}+\|y(0)\|_{\mathcal{C}(\bar{\Omega})} .
$$

Then $\mathcal{Y}$ is a Banach space, continuously embedded into $\mathcal{C}(\bar{Q})$ (see [1] for example).

Our main task is to show the existence of regular Lagrange multipliers for the state-constraints (Ib). The space for these multipliers depends on how the inequalities are considered. We might define the inequalities in the space $L^{\infty}(Q) \times L^{\infty}(\Sigma)$, since $u$ and $v$ are bounded and measurable. In this case, the multipliers have to be defined in the associated dual space. We aim to avoid this space of nonregular multipliers for several well known reasons. Let us briefly sketch the main idea we have in mind. Introduce the Lagrange function $\mathcal{L}: \mathcal{Y} \times L^{\infty}(Q) \times L^{\infty}(\Sigma) \times W(0, T) \times L^{\infty}(Q) \times L^{\infty}(\Sigma) \rightarrow \mathbb{R}$

$$
\begin{aligned}
\mathcal{L}\left(y, u, v, p, \mu_{1}, \mu_{2}\right)= & J(y, u, v)-\int_{Q}\left[y_{t}+A y+d(y)-u\right] p d x d t-\int_{\Sigma}\left[\partial_{\nu_{A}} y+b(y)-v\right] p d \sigma d t \\
& +\int_{Q}\left(u-c_{Q}-\beta_{Q} y\right) \mu_{1} d x d t+\int_{\Sigma}\left(v-c_{\Sigma}-\beta_{\Sigma} y\right) \mu_{2} d \sigma d t
\end{aligned}
$$

$(d \sigma=d \sigma(x)$ denotes the surface measure on $\Gamma)$. In this way, the mixed pointwise control-state-constraints are eliminated by multipliers $\mu_{1}, \mu_{2}$, while the control constraints remain unchanged. Let $(\bar{y}, \bar{u}, \bar{v})$ be a locally optimal triplet for $(\mathcal{P})$. We introduce the control sets

$$
U_{a d}=\left\{u \in L^{\infty}(Q) \mid u \geq 0 \text { a.e. }\right\}, \quad V_{a d}=\left\{v \in L^{\infty}(\Sigma) \mid v \geq 0 \text { a.e. }\right\}
$$

in case I and

$$
U_{a d}=\left\{u \in L^{\infty}(Q) \mid u \in\left[0, u_{b}\right] \text { a.e. }\right\}, \quad V_{a d}=\left\{v \in L^{\infty}(\Sigma) \mid u \in\left[0, v_{b}\right] \text { a.e. }\right\}
$$

in case II. Tacitly assuming that we shall be able to find regular multipliers $\mu_{1}, \mu_{2}$, the first order necessary optimality conditions for $(\bar{y}, \bar{u}, \bar{v})$ read

$$
\begin{array}{lll}
\mathcal{L}_{y}\left(\bar{y}, \bar{u}, \bar{v}, p, \mu_{1}, \mu_{2}\right) y & =0 \quad \forall y \in\{y \in \mathcal{Y} \mid y(0)=0\} \\
\mathcal{L}_{u}\left(\bar{y}, \bar{u}, \bar{v}, p, \mu_{1}, \mu_{2}\right)(u-\bar{u}) \geq 0 \quad \forall u \in U_{a d} \\
\mathcal{L}_{v}\left(\bar{y}, \bar{u}, \bar{v}, p, \mu_{1}, \mu_{2}\right)(v-\bar{v}) \geq 0 \quad \forall v \in V_{a d}
\end{array}
$$

Moreover, the complementary slackness conditions

$$
\mu_{1}\left(\bar{u}-c_{Q}-\beta_{Q} \bar{y}\right)=0 \text { a.e. on } Q \quad \text { and } \quad \mu_{2}\left(\bar{v}-c_{\Sigma}-\beta_{\Sigma} \bar{y}\right)=0 \text { a.e. on } \Sigma
$$

must be satisfied. 
From these relations we obtain the first order optimality system consisting of the state-equation, all inequality constraints, the adjoint equation

$$
\left\{\begin{aligned}
-p_{t}+A p+d_{y}(\bar{y}) p & =\alpha_{Q}\left(\bar{y}-z_{Q}\right)-\beta_{Q} \mu_{1} & & \text { in } Q \\
\partial_{\nu_{A}} p+b_{y}(\bar{y}) p & =\alpha_{\Sigma}\left(\bar{y}-z_{\Sigma}\right)-\beta_{\Sigma} \mu_{2} & & \text { on } \Sigma, \\
p(T) & =\alpha_{\Omega}\left(\bar{y}(T)-z_{\Omega}\right) & & \text { in } \Omega,
\end{aligned}\right.
$$

the variational inequalities

$$
\begin{aligned}
& \int_{Q}\left(\alpha_{u} \bar{u}+p+\mu_{1}\right)(u-\bar{u}) d x d t \geq 0 \quad \forall u \in U_{a d} \\
& \int_{\Sigma}\left(\alpha_{v} \bar{v}+p+\mu_{2}\right)(v-\bar{v}) d \sigma d t \geq 0 \quad \forall v \in V_{a d}
\end{aligned}
$$

the complementary slackness conditions, and the nonnegativity conditions

$$
\mu_{1}(x, t) \geq 0, \mu_{2}(x, t) \geq 0
$$

to be fulfilled for almost every $(x, t)$. Let $\left(p, \mu_{1}, \mu_{2}\right) \in W(0, T) \times L^{\infty}(Q) \times L^{\infty}(\Sigma)$ be any triplet satisfying, together with $(\bar{y}, \bar{u}, \bar{v})$, the optimality conditions formulated above. Then the function $p$ is said to be an adjoint state, and the $\mu_{i}$ are called Lagrange multipliers associated with $(\bar{y}, \bar{u}, \bar{v})$.

We shall derive conditions ensuring the existence of Lagrange multipliers $\mu_{1}, \mu_{2}$ in $L^{\infty}(Q) \times L^{\infty}(\Sigma)$. The idea is as follows: a constraint qualification is formulated for the state-constraints in $L^{\infty}$, where the natural cone of nonnegative functions has a nonempty interior. Therefore, this condition has a good chance to be satisfied. Next a known result by Zowe and Kurcyusz [7] yields that $(\bar{y}, \bar{u}, \bar{v})$ is a solution of the associated linearized problem

$$
\left(\mathcal{P}^{\ell}\right) \quad \min J^{\prime}(\bar{y}, \bar{u}, \bar{v})(y, u, v)
$$

subject to the linearized equation

$$
\left\{\begin{aligned}
y_{t}+A y+d(\bar{y})+d_{y}(\bar{y})(y-\bar{y}) & =u \\
\partial_{\nu_{A}} y+b(\bar{y})+b_{y}(\bar{y})(y-\bar{y}) & =v \\
y(x, 0) & =y_{o}(x)
\end{aligned}\right.
$$

and to the (linear) constraints $(y, u, v) \in C .\left(\mathcal{P}^{\ell}\right)$ is a linear continuous programming problem of bottleneck type, for which the duality theory of linear programming holds. In [2], we have proven existence of bounded and measurable solutions $\mu_{i} \geq 0$ for the associated dual problem. These solutions are Lagrange multipliers for $\left(\mathcal{P}^{\ell}\right)$. Then it is easy to verify that they are multipliers for $(\mathcal{P})$, too.

\section{Existence of optimal solutions to $\left(\mathcal{P}_{i}\right)$}

\subsection{Existence of a solution to the state-equation}

The following theorem ensures the existence and uniqueness of the state $y$ associated with $(u, v)$.

Theorem 2.1. For each pair $(u, v) \in L^{q}(Q) \times L^{s}(\Sigma)$, equation (1.1) has a unique solution $y=y(u, v) \in \mathcal{Y}$. Moreover

$$
\|y(u, v)\|_{W(0, T)}+\|y(u, v)\|_{\mathcal{C}(\bar{Q})} \leq c\left(1+\|u\|_{L^{q}(Q)}+\|v\|_{L^{s}(\Sigma)}+\left\|y_{o}\right\|_{\mathcal{C}(\bar{\Omega})}\right) .
$$


Proof. See [4] Theorem 4.13 or [5] Theorem 3.1. We should mention that the assumptions of [4] or [5] are implied by (A1-A2), since $u$ and $v$ appear here in a very simple linear form. The main assumption is here (A2), (i-ii).

Thanks to this result, the operator $\mathcal{T}$,

$$
\begin{aligned}
\mathcal{T}: \mathcal{Y} \times L^{q}(Q) \times L^{s}(\Sigma) & \rightarrow \quad L^{q}(Q) \times L^{s}(\Sigma) \times \mathcal{C}(\bar{\Omega}) \\
(y, u, v) & \mapsto\left[\begin{array}{c}
y_{t}+A y+d(y)-u \\
\partial_{\nu_{A}} y+b(y)-v \\
y(0)-y_{o}
\end{array}\right]
\end{aligned}
$$

is of class $\mathcal{C}^{1}$. We shall regard $\mathcal{T}$ also as an operator from $\mathcal{Y}_{\infty} \times L^{\infty}(Q) \times L^{\infty}(\Sigma)$ to $L^{\infty}(Q) \times L^{\infty}(\Sigma) \times \mathcal{C}(\bar{\Omega})$, where $\mathcal{Y}_{\infty}$ is defined analogously to $\mathcal{Y}$ with $s$ and $q$ substituted by $\infty$.

\subsection{Boundedness properties for feasible elements}

In the sequel, the set of $(y, u, v) \in \mathcal{Y} \times L^{\infty}(Q) \times L^{\infty}(\Sigma)$ satisfying all constraints of $(\mathcal{P})$ including the state equation, is said to be the feasible set. We assume this feasible set to be non empty. This is true, for example, if we assume that $y_{o} \geq 0$ and $d(\cdot, \cdot, 0)=b(\cdot, \cdot, 0)=0$ : indeed the triplet $(y, 0,0)$ is feasible, where $y$ is the solution to (1.1) with $u=0$ and $v=0$ (that is $\mathcal{T}(y, 0,0)=(0,0,0)$ ). This is a direct consequence of Theorem 2.3 here below, which implies $y$ to be non-negative by comparison with the null function $\tilde{y}$ which is solution to $\mathcal{T}(\tilde{y}, 0,0)=\left(0,0,-y_{0}\right)$.

We shall prove in this section that the feasible set is uniformly bounded for both the problems $\left(\mathcal{P}_{i}\right), i=1,2$. Let us first derive some auxiliary results.

Proposition 2.2 (Comparison principle for the linear equation). Suppose that functions $a, u \in L^{q}(Q), \alpha, v \in$ $L^{s}(\Sigma)$, and $y_{o} \in \mathcal{C}(\bar{\Omega})$ are given, such that

$$
a(x, t) \geq c_{o} \text { a.e. in } Q, \alpha(x, t) \geq c_{o} \text { a.e. on } \Sigma
$$

holds with some constant $c_{o} \in \mathbb{R}$. Let $y \in \mathcal{C}(\bar{Q})$ be the unique solution of the linear parabolic equation

$$
\left\{\begin{aligned}
y_{t}+A y+a y & =u \\
\partial_{\nu_{A}} y+\alpha y & =v \\
y(x, 0) & =y_{o}(x) .
\end{aligned}\right.
$$

If $u, v$ and $y_{o}$ are nonpositive a.e. on their domains of definition, then $y \leq 0$ holds everywhere in $\bar{Q}$.

This result can be found in [5], Proposition 3.2. It holds even for $y_{o} \in L^{2}(\Omega)$ in the sense that $y$ and its trace are almost everywhere nonpositive.

Now we are able to derive a comparison principle for the nonlinear state-equation. If $u$ is a bounded and measurable function being nonnegative almost everywhere on its domain of definition, then we shall write for convenience $u \geq 0$.

Theorem 2.3 (Comparison principle for the state-equation). Suppose $u_{i} \in L^{q}(Q)$ and $v_{i} \in L^{s}(\Sigma), i=1,2$. Let $y_{i}$ be the corresponding states, that is $\mathcal{T}\left(y_{1}, u_{1}, v_{1}\right)=\left(0,0, y_{1, o}\right)$ and $\mathcal{T}\left(y_{2}, u_{2}, v_{2}\right)=\left(0,0, y_{2, o}\right)$. If $u_{1} \geq$ $u_{2}, v_{1} \geq v_{2}$ and $y_{1, o} \geq y_{2, o}$, then $y_{1} \geq y_{2}$.

Proof. We know that the states $y_{i}$ belong to $\mathcal{C}(\bar{Q})$. Since $d$ is of class $\mathcal{C}^{1}$ with respect to $y$, we may use the mean value theorem so that

$$
\left.d\left(x, t, y_{2}(x, t)\right)-d\left(x, t, y_{1}(x, t)\right)=d_{y}\left(x, t, y_{\theta}(x, t)\right) \dot{(} y_{2}(x, t)-y_{1}(x, t)\right)
$$


where $y_{\theta}(x, t)=y_{1}(x, t)+\theta(x, t)\left(y_{2}(x, t)-y_{1}(x, t)\right)$, and the function $\theta \in(0,1)$ can be taken measurable. As $y_{i}$ is continuous and $d_{y}$ is a Carathéodory function, it is easy to conclude that $a(x, t)=d_{y}\left(x, t, y_{\theta}(x, t)\right)$ is bounded and measurable in $Q$. In particular, $a$ is bounded from below by (A2). The same argument applies to $b$, setting $\alpha(x, t)=b_{y}\left(x, t, y_{\theta}(x, t)\right)$ on $\Sigma$. In view of this, $y_{2}-y_{1}$ is the solution of the linear equation

$$
\left\{\begin{aligned}
\left(y_{2}-y_{1}\right)_{t}+A\left(y_{2}-y_{1}\right)+a\left(y_{2}-y_{1}\right) & =u_{2}-u_{1} \\
\partial_{\nu_{A}}\left(y_{2}-y_{1}\right)+\alpha\left(y_{2}-y_{1}\right) & =v_{2}-v_{1} \\
\left(y_{2}-y_{1}\right)(x, 0) & =\left(y_{o}+y_{2, o}\right)-\left(y_{o}+y_{1, o}\right)=y_{2, o}-y_{1, o} .
\end{aligned}\right.
$$

We complete the proof by Proposition 2.2.

Corollary 2.4. Let all assumptions of the previous theorem be satisfied. Then its conclusion remains true, if $(d, b)$ is replaced by $\left(d-\beta_{Q} y, b-\beta_{\Sigma} y\right)$ in the nonlinear state-equation.

Proof. This is obvious, since $\left(d-\beta_{Q} y\right)_{y}=d_{y}-\beta_{Q}$ belongs to $L^{\infty}(Q)$ and therefore is bounded from below. The same argument holds for $b$.

To prove the next theorem, we consider the state function $y=\hat{y}$, which corresponds to controls $u$ and $v$ acting at their upper limits, that is, $u=c_{Q}+\beta_{Q} y$ and $v=c_{\Sigma}+\beta_{\Sigma} y$. Inserting these controls in the equation of state, we arrive at the system

$$
\left\{\begin{aligned}
\hat{y}_{t}+A \hat{y}+d(\hat{y})-\beta_{Q} \hat{y} & =c_{Q} \\
\partial_{\nu_{A}} \hat{y}+b(\hat{y})-\beta_{\Sigma} \hat{y} & =c_{\Sigma} \\
\hat{y}(x, 0) & =y_{o}(x)
\end{aligned}\right.
$$

According to Theorem 2.1, this system admits a unique solution $\hat{y} \in \mathcal{C}(\bar{Q})$.

Theorem 2.5. The feasible sets of $\left(\mathcal{P}_{i}\right), i=1,2$, are bounded in $\mathcal{C}(\bar{Q}) \times L^{\infty}(Q) \times L^{\infty}(\Sigma)$.

Proof. (i) For $C_{2}$, the result is obvious: here, the admissible controls $u(x, t)$ and $v(x, t)$ belong to the uniformly bounded sets $\left[0, u_{b}\right]$ and $\left[0, v_{b}\right]$, respectively. Therefore, relation $(2.1)$ yields that all associated states $y=y(u, v)$ are uniformly bounded as well.

(ii) The situation is more interesting for $C_{1}$ : Let $u \geq 0$ and $v \geq 0$ satisfy, together with $y$, the state constraints. Then $u=c_{Q}-\delta_{Q}+\beta_{Q} y, v=c_{\Sigma}-\delta_{\Sigma}+\beta_{\Sigma} y$, where $\delta_{Q} \geq 0, \delta_{\Sigma} \geq 0$. Therefore,

$$
\left\{\begin{aligned}
y_{t}+A y+d(y)-\beta_{Q} y & =c_{Q}-\delta_{Q} \leq c_{Q} \\
\partial_{\nu_{A}} y+b(y)-\beta_{\Sigma} y & =c_{\Sigma}-\delta_{\Sigma} \leq c_{\Sigma} \\
y(0) & =y_{o} .
\end{aligned}\right.
$$

Corollary 2.4 yields $y \leq \hat{y}$, hence $u \leq c_{Q}+\beta_{Q} y \leq c_{Q}+\beta_{Q} \hat{y}$ (notice that $\beta_{Q}$ is nonnegative). This shows that all admissible controls $u$ are uniformly bounded. The same holds for $v$. Thanks to the estimate (2.1), all $y$ are uniformly bounded, too.

Remark 2.6. A study of the proof reveals that the property $u \in L^{\infty}(Q), v \in L^{\infty}(\Sigma)$ was not needed to apply the comparison principle. To apply this principle, it is sufficient to have $u \in L^{q}(Q)$ and $v \in L^{s}(\Sigma)$. In this case, the uniform boundedness $u \leq c_{Q}+\beta_{Q} \hat{y}$ still holds. Therefore, we are justified to regard the sets $C_{i}$ in $L^{q}(Q) \times L^{s}(\Sigma)$. This will not change them in comparison with their former definition in $L^{\infty}(Q) \times L^{\infty}(\Sigma)$, and the statement of Theorem 2.5 remains valid.

We are mainly interested in necessary optimality conditions for optimal controls. This refers to locally optimal controls as well. Therefore, we are justified to assume that a pair of locally optimal controls is given. Nevertheless, we briefly discuss the existence of (globally) optimal controls, since this is an important information on the well-posedness of the problem. 
Theorem 2.7. Problem $\left(\mathcal{P}_{i}\right)(i=1,2)$ has at least one optimal solution $(\bar{y}, \bar{u}, \bar{v}) \in \mathcal{Y} \times L^{\infty}(Q) \times L^{\infty}(\Sigma)$.

Proof. We shall only briefly sketch the idea of the proof. Thanks to Remark 2.6, we are justified to view $C_{i}$ as a bounded set of $\mathcal{Y} \times L^{q}(Q) \times L^{s}(\Sigma)$. If $\left\{\left(y_{n}, u_{n}, v_{n}\right)\right\}$ is a minimizing sequence, we can therefore assume that $\left\{u_{n}-d\left(y_{n}\right)\right\}$ and $\left\{v_{n}-b\left(y_{n}\right)\right\}$ are weakly converging in $L^{q}$ and $L^{s}$, respectively. The linear solution mapping can be represented by an integral operator (variations of constants formula or use of a Green's function) see, for instance [6], which is known to be compact from $L^{q}(Q) \times L^{s}(\Sigma)$ to $\mathcal{C}(\bar{Q})$. Therefore, $\left\{y_{n}\right\}$ tends strongly to $\bar{y}$ in $\mathcal{C}(\bar{Q})$, hence also $d\left(y_{n}\right)$ to $d(\bar{y})$ and $b\left(y_{n}\right)$ to $b(\bar{y})$. Moreover, $u_{n} \rightarrow \bar{u}$ and $v_{n} \rightarrow \bar{v}$ (weakly). We obtain by

the continuity of the linear solution mapping that $(\bar{y}, \bar{u}, \bar{v})$ solves the nonlinear state-equation. Moreover, the lower semicontinuity of $J$ with respect to $(u, v)$ permits to show in a standard way the optimality of $(\bar{y}, \bar{u}, \bar{v})$.

\section{Linearization of $(\mathcal{P})$}

Let us write for short $w:=(y, v, u)$. In this section, we regard $\mathcal{T}$ as an operator defined in $\mathcal{Y}_{\infty}$ $\times L^{\infty}(Q) \times L^{\infty}(\Sigma)$. With this notation, problem $(\mathcal{P})$ admits the abstract form

$$
\min \{J(w) \mid \mathcal{T}(w)=0, w \in C\}
$$

where $\mathcal{T}$ is differentiable and the convex and closed set $C$ stands for $C_{1}$ or $C_{2}$. To formulate a corresponding regularity condition, we define the linearized cone of $C$ at $\bar{w}$,

$$
C(\bar{w})=\{\lambda(w-\bar{w}) \mid \lambda \geq 0, w=(y, u, v) \in C\}
$$

The following general result is known for the linearization of $(\mathcal{P})$ at the optimal point $[6,7]$ :

Theorem 3.1. Assume that the regularity assumption

$$
\mathcal{T}^{\prime}(\bar{w}) \cdot C(\bar{w})=L^{\infty}(Q) \times L^{\infty}(\Sigma) \times C(\bar{\Omega}),
$$

is satisfied at $\bar{w}$. Then $\bar{w}$ is solution of the linearized problem

$$
\left\{\begin{array}{l}
\min J^{\prime}(\bar{w}) \cdot w \\
\mathcal{T}^{\prime}(\bar{w}) \cdot(w-\bar{w})=0 \\
w \in C
\end{array}\right.
$$

Remark 3.2. The original result of [7] states that

$$
J^{\prime}(\bar{w}) \cdot h \geq 0 \quad \forall h \in C(\bar{w}): \mathcal{T}^{\prime}(\bar{w}) h=0
$$

We have $h=\lambda(w-\bar{w})$. Inserting this for $\lambda=1$, the inequality $J^{\prime}(\bar{w}) \cdot(w-\bar{w}) \geq 0$ follows, so that $\bar{w}$ attains the minimal value.

In our case, the sets $C=C_{i}, i=1,2$, have obviously a nonempty interior in the space $\mathcal{C}(\bar{Q}) \times L^{\infty}(Q) \times L^{\infty}(\Sigma)$. Therefore it makes sense to assume the following stronger regularity condition, which is known to be sufficient for (3.1) to hold [6]: $\mathcal{T}$ is surjective and

$$
\left\{\begin{array}{c}
\exists(\tilde{y}, \tilde{u}, \tilde{v}) \in \operatorname{int} C \text { such that } \\
\mathcal{T}^{\prime}(\bar{y}, \bar{u}, \bar{v})(\tilde{y}-\bar{y}, \tilde{u}-\bar{u}, \tilde{v}-\bar{v})=0 .
\end{array}\right.
$$


Here, int $C$ denotes the $L^{\infty}$-interior and $\tilde{y}$ belongs to $\mathcal{Y}_{\infty}$. Since $\mathcal{T}$ is surjective, we have to find a triplet $(\tilde{y}, \tilde{u}, \tilde{v})$ of $\mathcal{Y} \times L^{\infty}(Q) \times L^{\infty}(\Sigma)$ such that

$$
\left\{\begin{aligned}
(\tilde{y}-\bar{y})_{t}+A(\tilde{y}-\bar{y})+d_{y}(\bar{y})(\tilde{y}-\bar{y}) & =\tilde{u}-\bar{u} \\
\partial_{\nu_{A}}(\tilde{y}-\bar{y})+b_{y}(\bar{y})(\tilde{y}-\bar{y}) & =\tilde{v}-\bar{v} \\
\tilde{y}(0)-\bar{y}(0) & =0
\end{aligned}\right.
$$

and

$$
\begin{aligned}
& \varepsilon \leq \tilde{u}(x, t) \leq c_{Q}(x, t)-\varepsilon+\beta_{Q}(x, t) \tilde{y}(x, t) \\
& \varepsilon \leq \tilde{v}(x, t) \leq c_{\Sigma}(x, t)-\varepsilon+\beta_{\Sigma}(x, t) \tilde{y}(x, t)
\end{aligned}
$$

holds a.e. on $Q$ and $\Sigma$, respectively, where $\varepsilon$ is positive, for the case where $C_{i}=C_{1}$. When $C_{i}=C_{2}$, we demand in addition

$$
\begin{aligned}
& \tilde{u}(x, t) \leq u_{b}(x, t)-\varepsilon \\
& \tilde{v}(x, t) \leq v_{b}(x, t)-\varepsilon .
\end{aligned}
$$

We shall verify these conditions under the following very natural assumption:

(A3) There is a positive constant $\delta$ such that

$$
\begin{aligned}
& c_{Q}(x, t)+\beta_{Q}(x, t) \bar{y}(x, t) \geq \delta \\
& c_{\Sigma}(x, t)+\beta_{\Sigma}(x, t) \bar{y}(x, t) \geq \delta .
\end{aligned}
$$

In other words, the sets $\left\{u \in L^{\infty}(Q) \mid 0 \leq u \leq c_{Q}+\beta_{Q} \bar{y}\right\}$ and $\left\{v \in L^{\infty}(\Sigma) \mid 0 \leq v \leq c_{\Sigma}+\beta_{\Sigma} \bar{y}\right\}$ (defined upon $\bar{y}$ ) are assumed to have a nonempty interior. In particular, this assumption is satisfied, if $c_{Q}$ and $c_{\Sigma}$ are bounded from below by a positive constant (a condition being assumed in this paper) and if $\bar{y}$ is known to be nonnegative (which is the case if $y_{o}$ is nonnegative).

To verify that (A3) implies (3.2), let us discuss case II. It is technically more difficult than case I, which can be proved by an evident modification. We introduce the "maximal linearized solution" $\hat{y}$ by inserting $\hat{u}:=\min \left\{u_{b}, c_{Q}+\beta_{Q} \hat{y}\right\}, \hat{v}:=\min \left\{v_{b}, c_{\Sigma}+\beta_{\Sigma} \hat{y}\right\}$ in the linearized equation (1.9). In this way, $\hat{y}$ is defined by

$$
\left\{\begin{aligned}
\hat{y}_{t}+A \hat{y}+d(\bar{y})+d_{y}(\bar{y})(\hat{y}-\bar{y}) & =\min \left\{u_{b}, c_{Q}+\beta_{Q} \hat{y}\right\} \quad(=\hat{u}) \\
\partial_{\nu_{A}} \hat{y}+b(\bar{y})+b_{y}(\bar{y})(\hat{y}-\bar{y}) & =\min \left\{v_{b}, c_{\Sigma}+\beta_{\Sigma} \hat{y}\right\} \quad(=\hat{v}) \\
\hat{y}(0) & =y_{o} .
\end{aligned}\right.
$$

The existence of the solution $\hat{y}$ to (3.6) and its uniqueness can be shown by the same technique, which was used to prove Lemma 2.6 in [2]. We avoid this proof, since the implication (A3) $\Rightarrow(3.2)$ does not play a central role in our paper.

Lemma 3.3. The relation $\hat{y}(x, t) \geq \bar{y}(x, t)$ holds for all $(x, t) \in \bar{Q}$.

Proof. Define $Q_{1}$ by

$$
Q_{1}=\left\{(x, t) \in Q \mid u_{b}<c_{Q}+\beta_{Q} \hat{y}\right\}
$$

and put $Q_{2}=Q \backslash Q_{1}$. On $Q_{1}$ we have $\hat{u}=u_{b}$, while $\hat{u}=c_{Q}+\beta_{Q} \hat{y}$ holds on $Q_{2}$. Completely analogous, we introduce sets $\Sigma_{i}, i=1,2$, for $\hat{v}$. Define $\hat{c}_{Q}, \hat{\beta}_{Q}$ by $\hat{c}_{Q}=u_{b}, \hat{\beta}_{Q}=0$ on $Q_{1}, \hat{c}_{Q}=c_{Q}, \hat{\beta}_{Q}=\beta_{Q}$ on $Q_{2}$. Then $\hat{u}=\hat{c}_{Q}+\hat{\beta}_{Q} \hat{y}$ everywhere on $Q$. Analogously, $\hat{v}=\hat{c}_{\Sigma}+\hat{\beta}_{\Sigma} \hat{y}$ is obtained on $\Sigma=\Sigma_{1} \cup \Sigma_{2}$. In view of this,

$$
\begin{array}{ll}
\bar{u} \leq \hat{c}_{Q}+\hat{\beta}_{Q} \bar{y} & \text { on } Q \\
\hat{u}=\hat{c}_{Q}+\hat{\beta}_{Q} \hat{y} & \text { on } Q .
\end{array}
$$


The function $\bar{y}(x, t)$ satisfies

$$
\left\{\begin{aligned}
\bar{y}_{t}+A \bar{y}+d(\bar{y}) & =\bar{u}=\hat{c}_{Q}+\hat{\beta}_{Q} \bar{y}-\delta_{Q} \\
\partial_{\nu_{A}} \bar{y}+b(\bar{y}) & =\bar{v}=\hat{c}_{\Sigma}+\hat{\beta}_{\Sigma} \bar{y}-\delta_{\Sigma} \\
\bar{y}(0) & =y_{o}
\end{aligned}\right.
$$

where $\delta_{Q}$ and $\delta_{\Sigma}$ are nonnegative. Subtracting (3.7) from (3.6) we find

$$
\left\{\begin{aligned}
(\hat{y}-\bar{y})_{t}+A(\hat{y}-\bar{y})+d_{y}(\bar{y})(\hat{y}-\bar{y})-\hat{\beta}_{Q}(\hat{y}-\bar{y}) & =\delta_{Q} \\
\partial_{\nu_{A}}(\hat{y}-\bar{y})+b_{y}(\bar{y})(\hat{y}-\bar{y})-\hat{\beta}_{\Sigma}(\hat{y}-\bar{y}) & =\delta_{\Sigma} \\
\hat{y}(0)-\bar{y}(0) & =0 .
\end{aligned}\right.
$$

The comparison principle yields $\hat{y}-\bar{y} \geq 0$.

Theorem 3.4. Assumption (A3) implies the regularity condition (3.2).

Proof. To construct $(\tilde{y}, \tilde{u}, \tilde{v})$, take a constant $\lambda<1$ close to 1 and define $\tilde{y}$ by

$$
\left\{\begin{aligned}
\tilde{y}_{t}+A \tilde{y}+d(\bar{y})+d_{y}(\bar{y})(\tilde{y}-\bar{y}) & =\min \left\{\lambda u_{b}, \lambda c_{Q}+\beta_{Q} \tilde{y}\right\} \quad(=: \tilde{u}) \\
\partial_{\nu_{A}} \tilde{y}+b(\bar{y})+b_{y}(\bar{y})(\tilde{y}-\bar{y}) & =\min \left\{\lambda v_{b}, \lambda c_{\Sigma}+\beta_{\Sigma} \tilde{y}\right\} \quad(=: \tilde{v}) \\
\tilde{y}(0) & =y_{o}
\end{aligned}\right.
$$

Then

$$
\left\{\begin{aligned}
(\tilde{y}-\bar{y})_{t}+A(\tilde{y}-\bar{y})+d_{y}(\bar{y})(\tilde{y}-\bar{y}) & =\tilde{u}-\bar{u} \\
\partial_{\nu_{A}}(\tilde{y}-\bar{y})+b_{y}(\bar{y})(\tilde{y}-\bar{y}) & =\tilde{v}-\bar{v} \\
\tilde{y}(0)-\bar{y}(0) & =0
\end{aligned}\right.
$$

and the equation $\mathcal{T}^{\prime}(\bar{w})(\tilde{w}-\bar{w})=0$ is satisfied. By continuity, $\tilde{y}$ tends uniformly in $\bar{Q}$ towards $\hat{y}$, as $\lambda$ tends to 1 .

We briefly explain this: let $y_{1}$ denote the solution of (3.8) for $\lambda=1$, and define $u_{1}$ (resp. $\tilde{u}$ ) by the equations $u_{1}=\min \left\{u_{b}, c_{Q}+\beta_{Q} y_{1}\right\}\left(\operatorname{resp} . \tilde{u}=\min \left\{\lambda u_{b}, \lambda c_{Q}+\beta_{Q} \tilde{y}\right\}\right)$. Then, by $\min (a, b)=\frac{1}{2}(a+b-|a-b|)$,

$$
\begin{aligned}
u_{1} & =\frac{1}{2}\left(u_{b}+c_{Q}+\beta_{Q} y_{1}-\left|u_{b}-c_{Q}-\beta_{Q} y_{1}\right|\right) \\
\tilde{u} & =\frac{1}{2}\left(\lambda\left(u_{b}+c_{Q}\right)+\beta_{Q} \tilde{y}-\left|\lambda\left(u_{b}-c_{Q}\right)-\beta_{Q} \tilde{y}\right|\right),
\end{aligned}
$$

and a simple estimation gives $\left|u_{1}-\tilde{u}\right| \leq c(1-\lambda)+c\left|y_{1}-\tilde{y}\right|$ on $Q$. An estimate for $\left|v_{1}-\tilde{v}\right|$ on $\Sigma$ can be derived in the same way. Finally, we consider the equation for $y_{1}-\tilde{y}$. Its right hand sides are estimated by $c(1-\lambda)+\left|y_{1}-\tilde{y}\right|$, and the property $\left|y_{1}-\tilde{y}\right| \rightarrow 0$ follows from an application of the comparison principle.

Now we return to the main line of proof and pass to the limit $\lambda \rightarrow 1$. Then

$$
\tilde{u}=\min \left\{\lambda u_{b}, \lambda c_{Q}+\beta_{Q} \tilde{y}\right\} \geq \frac{\delta}{2} \text { and } \tilde{v}=\min \left\{\lambda v_{b}, \lambda c_{\Sigma}+\beta_{\Sigma} \tilde{y}\right\} \geq \frac{\delta}{2}
$$

follows from assumption (A3) and Lemma 3.3 for $\lambda$ sufficiently close to 1 . Moreover,

$$
\begin{aligned}
& \tilde{u}=\min \left\{\lambda u_{b}, \lambda c_{Q}+\beta_{Q} \tilde{y}\right\}<\min \left\{u_{b}, c_{Q}+\beta_{Q} \tilde{y}\right\}, \\
& \tilde{v}=\min \left\{\lambda v_{b}, \lambda c_{\Sigma}+\beta_{\Sigma} \tilde{y}\right\}<\min \left\{v_{b}, c_{\Sigma}+\beta_{\Sigma} \tilde{y}\right\} .
\end{aligned}
$$

Altogether, the last relations show that $(\tilde{y}, \tilde{u}, \tilde{v})$ belongs to the $L^{\infty}$-interior of $C_{1}$. 
The proof of case I can be performed analogously by the formal setting $u_{b}=\infty, v_{b}=\infty$. In this case, the arguments are even simpler.

From now on, we assume that (A3) is fulfilled so that Theorem 3.1 is applicable. The linearized problem has been defined in an abstract setting. Inserting the concrete expressions for $J^{\prime}$ and $\mathcal{T}^{\prime}$, it admits the following explicit form:

$$
\begin{aligned}
& \min \quad \alpha_{Q} \int_{Q}\left(\bar{y}-z_{Q}\right) \cdot y d x d t+\alpha_{\Omega} \int_{\Omega}\left(\bar{y}(T)-z_{\Omega}\right) \cdot y(T) d x+ \\
& \quad \alpha_{\Sigma} \int_{\Sigma}^{\ell}\left(\bar{y}-z_{\Sigma}\right) \cdot y d \sigma d t+\alpha_{u} \int_{Q} \bar{u} \cdot u d x d t+\alpha_{v} \int_{\Sigma} \bar{v} \cdot v d \sigma d t \\
& \text { subject to } \\
& \qquad \begin{aligned}
y_{t}+A y+d_{y}(\bar{y}) y & =u+d_{y}(\bar{y}) \bar{y}-d(\bar{y}) \\
\partial_{\nu_{A}} y+b_{y}(\bar{y}) y & =v+b_{y}(\bar{y}) \bar{y}-b(\bar{y}) \\
y(0) & =y_{o}
\end{aligned} \\
& \left\{\begin{aligned}
(y, u, v) & \in C_{i} .
\end{aligned}\right.
\end{aligned}
$$

For convenience, we set

$$
\bar{\omega}_{Q}:=d(\bar{y})-d_{y}(\bar{y}) \cdot \bar{y} \in L^{\infty}(Q), \bar{\omega}_{\Sigma}:=b(\bar{y})-b_{y}(\bar{y}) \cdot \bar{y} \in L^{\infty}(\Sigma),
$$

and

$$
\begin{aligned}
& a_{Q}:=-\alpha_{Q}\left[\bar{y}-z_{Q}\right] \in L^{\infty}(Q), a_{\Omega}:=-\alpha_{\Omega}\left[\bar{y}(T)-z_{\Omega}\right] \in L^{\infty}(\Omega), \\
& a_{\Sigma}:=-\alpha_{\Sigma}\left[\bar{y}-z_{\Sigma}\right] \in L^{\infty}(\Sigma), a_{u}:=-\alpha_{u} \bar{u} \in L^{\infty}(Q), a_{v}:=-\alpha_{v} \bar{v} \in L^{\infty}(\Sigma),
\end{aligned}
$$

so that $\left(\mathcal{P}_{i}^{\ell}\right)$ can be written as

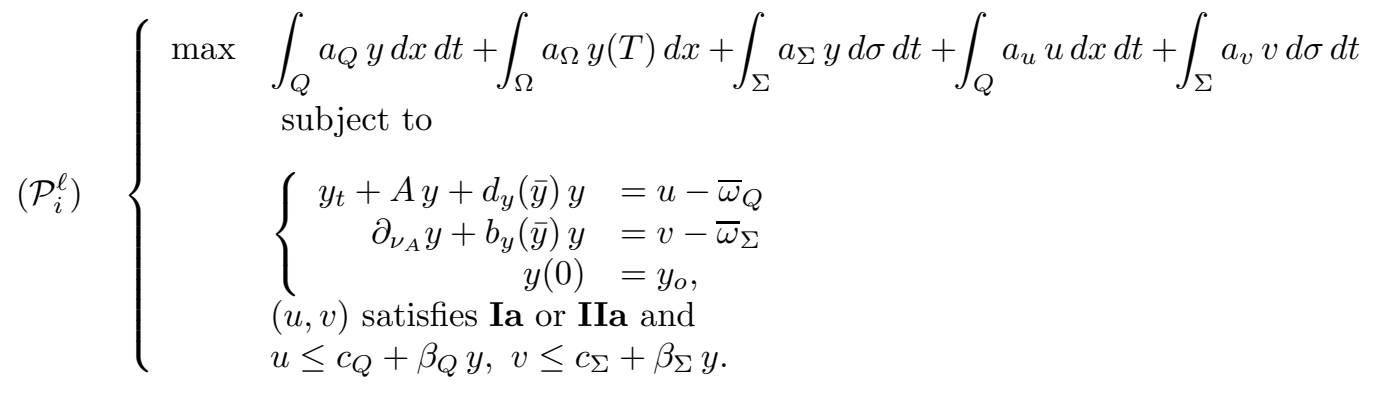

This kind of linear control problems has been studied in our paper [2].

\section{Existence of Regular Lagrange multipliers}

Let us assume from now on that $\Gamma$ and the coefficients of $A$ are sufficiently smooth to ensure the existence of a Green's function $G=G(x, \xi, t)$ associated with the linearized partial differential equation. This assumption can certainly be avoided. However, we want to directly apply our results of [2], where all main theorems were proved on using Green's functions. A study of our technique in [2] reveals that the theory can be developed also for weak solutions satisfying comparison principles. We shall not discuss this generalization here. The solution 
$y$ of (3.10) has the integral representation

$$
\begin{aligned}
y(x, t)= & \int_{\Omega} G(x, \xi, t) y_{o}(\xi) d \xi+\int_{0}^{t} \int_{\Omega} G(x, \xi, t-s)\left(u(\xi, s)-\bar{w}_{Q}(\xi, s)\right) d \xi d s \\
& +\int_{0}^{t} \int_{\Gamma} G(x, \xi, t-s)\left(v(\xi, s)-\bar{w}_{\Sigma}(\xi, s)\right) d \sigma(\xi) d s \\
= & y_{c}(x, t)+\int_{0}^{t} \int_{\Omega} G(x, \xi, t-s) u(\xi, s) d \xi d s+\int_{0}^{t} \int_{\Gamma} G(x, \xi, t-s) v(\xi, s) d \sigma(\xi) d s \\
= & y_{c}(x, t)+\eta(x, t)
\end{aligned}
$$

where $y_{c}$ denotes the constant part of $y$ corresponding to $\left(y_{o},-\bar{w}_{Q},-\bar{w}_{\Sigma}\right)$, and $\eta$ is the part associated with $(0, u, v)$. Inserting the expression (4.1) in $\left(\mathcal{P}_{i}^{\ell}\right)$, and using the Fubini theorem in the objective, we arrive at the following linear continuous programming problem of bottleneck type (primal problem):

$$
\max \int_{Q} a_{1}(x, t) u(x, t) d x d t+\int_{\Sigma} a_{2}(x, t) v(x, t) d \sigma d t
$$

subject to

$$
\begin{array}{ll}
u(x, t) \leq \bar{c}_{Q}(x, t) \quad & +\int_{0}^{t} \int_{\Omega} \beta_{Q}(x, t) G(x, \xi, t-s) u(\xi, s) d \xi d s \\
& +\int_{0}^{t} \int_{\Gamma} \beta_{Q}(x, t) G(x, \xi, t-s) v(\xi, s) d \sigma(\xi) d s \\
v(x, t) \leq \bar{c}_{\Sigma}(x, t) \quad & +\int_{0}^{t} \int_{\Omega} \beta_{\Sigma}(x, t) G(x, \xi, t-s) u(\xi, s) d \xi d s \\
& +\int_{0}^{t} \int_{\Gamma} \beta_{\Sigma}(x, t) G(x, \xi, t-s) v(\xi, s) d \sigma(\xi) d s \\
u(x, t) \geq 0, & \left(u(x, t) \leq u_{b}\right) \\
v(x, t) \geq 0, & \left(v(x, t) \leq v_{b}\right),
\end{array}
$$

where the upper bounds for the controls are only required in $\left(\mathcal{P}_{2}^{\ell}\right)$. The function $a_{1} \in L^{\infty}(Q)$ is defined by

$$
\begin{aligned}
a_{1}(x, t)= & \int_{\Omega} G(x, \xi, T-t) a_{\Omega}(\xi) d \xi+\int_{t}^{T} \int_{\Omega} G(x, \xi, s-t) a_{Q}(\xi, s) d \xi d s \\
& +\int_{t}^{T} \int_{\Gamma} G(x, \xi, s-t) a_{\Sigma}(\xi, s) d \sigma(\xi) d s+a_{u}(x, t)
\end{aligned}
$$

while $a_{2} \in L^{\infty}(\Sigma)$ has the same form with $a_{v}(x, t)$ substituted for the last item. Moreover, $\bar{c}_{Q}:=c_{Q}+\beta_{Q} y_{c}$, $\bar{c}_{\Sigma}:=c_{\Sigma}+\beta_{\Sigma} y_{c}$ were introduced. The two first inequality constraints of (4.2) admit the form

$$
u \leq \bar{c}_{Q}+\beta_{Q} \eta, \quad u \leq \bar{c}_{\Sigma}+\beta_{\Sigma} \eta
$$

The primal problem may have more than one solution. However, one of them is always $(\bar{u}, \bar{v})$. The associated state is $\bar{y}=y_{c}+\bar{\eta}$.

Let us define another auxiliary function $\psi \in \mathcal{Y}$ by

$$
\left\{\begin{aligned}
-\psi_{t}+A \psi+d_{y}(\bar{y}) \psi & =a_{Q} \\
\partial_{\nu_{A}} \psi+b_{y}(\bar{y}) \psi & =a_{\Sigma} \\
\psi(T)= & =a_{\Omega} .
\end{aligned}\right.
$$


Then we have $a_{1}=\psi+a_{u}$ and $a_{2}=\left.\psi\right|_{\Sigma}+a_{v}$, and the relation

$$
\begin{gathered}
\int_{Q} a_{1}(x, t) u(x, t) d x d t+\int_{Q} a_{2}(x, t) v(x, t) d \sigma d t \\
=\int_{\Omega} a_{\Omega} \eta(T) d x+\int_{Q} a_{Q} \eta d x d t+\int_{\Sigma} a_{\Sigma} \eta d \sigma d t+\int_{\Omega} a_{u} u d x d t+\int_{\Sigma} a_{v} v d \sigma d t
\end{gathered}
$$

is obtained integrating by parts on using $\psi$. The associated dual problem has the form

$$
\min \int_{Q}\left(\bar{c}_{Q} \mu_{1}+u_{b} \mu_{3}\right) d x d t+\int_{\Sigma}\left(\bar{c}_{\Sigma} \mu_{2}+v_{b} \mu_{4}\right) d \sigma d t
$$

subject to

$$
\begin{aligned}
\mu_{1}(x, t)+\mu_{3}(x, t) \geq a_{1}(x, t) \quad & +\int_{t}^{T} \int_{\Omega} G(x, \xi, s-t) \beta_{Q}(\xi, s) \mu_{1}(\xi, s) d \xi d s \\
& +\int_{t}^{T} \int_{\Gamma} G(x, \xi, s-t) \beta_{\Sigma}(\xi, s) \mu_{2}(\xi, s) d \sigma(\xi) d s, \\
\mu_{2}(x, t)+\mu_{4}(x, t) \geq \quad a_{2}(x, t) \quad & +\int_{t}^{T} \int_{\Omega} G(x, \xi, s-t) \beta_{Q}(\xi, s) \mu_{1}(\xi, s) d \xi d s \\
& +\int_{t}^{T} \int_{\Gamma} G(x, \xi, s-t) \beta_{\Sigma}(\xi, s) \mu_{2}(\xi, s) d \sigma(\xi) d s, \\
\mu_{i}(x, t) \geq \quad 0, & i=1, \ldots, 4 .
\end{aligned}
$$

In the case of $\left(\mathcal{P}_{1}\right)$, the functions $\mu_{3}, \mu_{4}$ do not appear: The associated dual problem is obtained by setting $\mu_{3}=\mu_{4}=0$. If we denote by $\varphi$ the solution of the system

$$
\left\{\begin{aligned}
-\varphi_{t}+A \varphi+d_{y}(\bar{y}) \varphi & =\beta_{Q} \mu_{1} \\
\partial_{\nu_{A}} \varphi+b_{y}(\bar{y}) \varphi & =\beta_{\Sigma} \mu_{2} \\
\varphi(T) & =0
\end{aligned}\right.
$$

then the constraints of the dual problem admit the simpler form

$$
\begin{aligned}
\mu_{1}+\mu_{3} & \geq a_{1}+\varphi \\
\mu_{2}+\mu_{4} & \geq a_{2}+\varphi \\
\mu_{i} & \geq 0, i=1, \ldots, 4 .
\end{aligned}
$$

In this way, the dual problem is seen to be a linear parabolic control problem with state-constraints. The theory in [2] yields the next theorem on the existence of at least one bounded and measurable optimal solution $\left(\bar{\mu}_{1}, \bar{\mu}_{2}, \bar{\mu}_{3}, \bar{\mu}_{4}\right)$ for the dual problem. The following assumption is needed:

(A4) The functions $\bar{c}_{Q}$ and $\bar{c}_{\Sigma}$ are nonnegative.

Remark 4.1. This assumption is satisfied, if $d_{y}(\bar{y}) \bar{y}-d(\bar{y}) \geq 0$, an analogous relation holds for $b$, and $y_{o}$ is nonnegative. This follows from (3.10) and the comparison principle. In particular, nonlinearities of the type $d(y)=y^{k}$ ( $k$ odd) or $d(y)=|y| y^{k-1}$ ( $k$ even) meet this assumption. Here, the comparison principle implies $\bar{y} \geq 0$, since $d$ is monotone non-decreasing.

Theorem 4.2. Suppose that (A3) is satisfied. Then the dual problem $\left(\mathcal{D}^{\ell}\right)$ admits at least one optimal solution $\left(\bar{\mu}_{1}, \bar{\mu}_{2}, \bar{\mu}_{3}, \bar{\mu}_{4}\right)$ that belongs to $L^{\infty}(Q) \times L^{\infty}(\Sigma) \times L^{\infty}(Q) \times L^{\infty}(\Sigma)$. 
The proof of the theorem was performed separately for the cases of boundary control and distributed control. However, it is clear that it can be extended to the general problem above (see the remarks in the last section of [2]). The idea of [2] is as follows: First of all, the feasible set of $\left(\mathcal{D}^{\ell}\right)$ is nonempty. This is a consequence of the duality relation $\max \left(\mathcal{P}^{\ell}\right)=\inf \left(\mathcal{D}^{\ell}\right)$, which follows from the simple structure of the constraints of $\left(\mathcal{P}^{\ell}\right)$. If a feasible $\left(\mu_{1}, \mu_{2}, \mu_{3}, \mu_{4}\right)$ is given such that $\mu_{1}(x, t)+\mu_{3}(x, t)$ is positive on some subset of $Q$, and $\mu_{1}(x, t)+\mu_{3}(x, t)>a_{1}(x, t)+\varphi(x, t)$ holds there, then we are able to diminish $\mu_{1}+\mu_{3}$ on this set, until the equality $\mu_{1}+\mu_{3}=\max \left\{a_{1}+\varphi, 0\right\}$ is achieved. In the same way, $\mu_{2}+\mu_{4}$ is handled. Here, we essentially use the positivity of $G(x, \xi, t)$. The value of the dual objective decreases, since $\bar{c}_{Q}, \bar{c}_{\Sigma}, u_{b}, v_{b}$ are positive. Finally, one is able to show that the infimum of $\left(\mathcal{D}^{\ell}\right)$ is attained in a uniformly bounded set. The solution is found by weak compactness.

We recall the complementary slackness conditions,

$$
\begin{array}{r}
\left(\bar{\mu}_{1}+\bar{\mu}_{3}-a_{1}-\varphi\right) \bar{u}=0, \bar{\mu}_{3}\left(\bar{u}-u_{b}\right)=0 \text { a.e in } Q \\
\left(\bar{\mu}_{2}+\bar{\mu}_{4}-a_{2}-\varphi\right) \bar{v}=0, \bar{\mu}_{4}\left(\bar{v}-v_{b}\right)=0 \text { a.e on } \Sigma \\
\bar{\mu}_{1}\left(\bar{u}-\bar{c}_{Q}-\beta_{Q} \bar{\eta}\right)=0 \\
\bar{\mu}_{2}\left(\bar{v}-\bar{c}_{\Sigma}-\beta_{\Sigma} \bar{\eta}\right)=0
\end{array}
$$

which are well known from the theory of linear continuous programming.

Theorem 4.3. If $\left(\bar{\mu}_{1}, \bar{\mu}_{2}, \bar{\mu}_{3}, \bar{\mu}_{4}\right)$ is a bounded and measurable optimal solution of the dual problem $\left(\mathcal{D}^{\ell}\right)$, then $\bar{\mu}_{1}$ and $\bar{\mu}_{2}$ are Lagrange multipliers for the (nonlinear) optimal control problem $(\mathcal{P})$, associated with the stateconstraints $u \leq c_{Q}+\beta_{Q} y$ and $v \leq c_{\Sigma}+\beta_{\Sigma} y$, respectively.

Proof. We define $p:=-(\psi+\varphi)$ and verify the optimality conditions (1.6-1.8): the adjoint equation (1.6) is easily obtained by adding the systems for $\psi$ and $\varphi$ with negative sign. Let us show the variational inequality (1.7). We find

$$
\begin{gathered}
\int_{Q}\left(\alpha_{u} \bar{u}+p+\bar{\mu}_{1}\right)(u-\bar{u}) d x d t=\int_{Q}\left(-a_{u}+p+\bar{\mu}_{1}\right)(u-\bar{u}) d x d t \\
=\int_{Q}\left(\psi-a_{1}+p+\bar{\mu}_{1}\right)(u-\bar{u}) d x d t=-\int_{Q}\left(a_{1}+\varphi-\bar{\mu}_{1}\right)(u-\bar{u}) d x d t \\
=\int_{Q}\left(a_{1}+\varphi-\bar{\mu}_{1}-\bar{\mu}_{3}\right) \bar{u} d x d t-\int_{Q}\left(a_{1}+\varphi-\bar{\mu}_{1}-\bar{\mu}_{3}\right) u d x d t+\int_{Q} \bar{\mu}_{3}\left(\bar{u}-u_{b}\right) d x d t-\int_{Q} \bar{\mu}_{3}\left(u-u_{b}\right) d x d t \geq 0 .
\end{gathered}
$$

The last inequality follows from the complementary slackness conditions $(4.8,4.9)$ for the terms containing $\bar{u}$, the nonnegativity of $\bar{\mu}_{1}, \bar{\mu}_{3}$, and from the inequality constraints $u \geq 0, u \leq u_{b}$. We have shown (1.7). The variational inequality (1.8) is verified in the same way. In view of the complementary slackness conditions, $\bar{\mu}_{1}, \bar{\mu}_{2}$ satisfy all properties of Lagrange multipliers. The complementary slackness condition (1.5) are obtained from $(4.10,4.11)$. For instance,

$$
0=\mu_{1}\left(\bar{u}-\bar{c}_{Q}-\beta_{Q} \bar{\eta}\right)=\mu_{1}\left(\bar{u}-c_{Q}-\beta_{Q}\left(y_{c}+\bar{\eta}\right)\right)=\mu_{1}\left(\bar{u}-c_{Q}-\beta_{Q} \bar{y}\right) .
$$

We have performed the proof for problem $\left(\mathcal{P}_{2}\right)$, the case of $\left(\mathcal{P}_{1}\right)$ is treated by $\bar{\mu}_{3}=0, \bar{\mu}_{4}=0$.

We are grateful to both the referees for their remarks, which helped us to essentially improve our paper.

\section{REFERENCES}

[1] M. Bergounioux and F. Tröltzsch, Optimality Conditions and Generalized Bang-Bang Principle for a State Constrained Semilinear Parabolic Problem. Num. Funct. Anal. Opt. 15 (1996) 517-537.

[2] M. Bergounioux and F. Tröltzsch, Optimal Control of Linear Bottleneck Problems. ESAIM: Cont. Optim. Cal. Var. 3 (1998) $235-250$. 
[3] E. Casas, Pontryagin's principle for state-constrained boundary control problems of semilinear parabolic equations. SIAM J. Control Optim. 35 (1997) 1297-1327.

[4] J.P. Raymond, Non Linear Boundary Control of Semilinear Parabolic Problems with Pointwise State Constraints. Discrete and Continuous Dynamical Systems 3 (1997) 341-370.

[5] J.P. Raymond and H. Zidani, Hamiltonian Pontryagin's Principles for Control Problems Governed by Semilinear Parabolic Equations. Appl. Math. Optim., to appear

[6] F. Tröltzsch, Optimality conditions for parabolic control problems and applications, Teubner Texte zur Mathematik, Teubner, Leipzig (1984).

[7] J. Zowe and S. Kurcyusz, Regularity and stability for the mathematical programming problem in Banach spaces. Appl. Math. Optim. 5 (1979) 49-62. 